\title{
Oppenheim's Problem and Some Inequalities Involving Bessel Functions and Dunkl Kernels
}

\author{
Frej Chouchene* \\ Department of Mathematics, University of Sousse, Higher School of Sciences and Technology of Hammam Sousse, \\ 4011 Hammam Sousse, Tunisia \\ *Corresponding author: frej.chouchene@essths.u-sousse.tn
}

Received January 01, 2018; Revised March 01, 2018; Accepted May 05, 2018

\begin{abstract}
In this paper, we establish some inequalities related to Oppenheim's problem for the real and imaginary parts of Dunkl kernels $\psi_{1}^{\alpha}, \alpha \geq-\frac{1}{2}$. In order to prove our main results, we present some new inequalities involving Bessel functions of the first kind. Refinements of inequalities for Bessel functions are also given.
\end{abstract}

Keywords: Oppenheim's problem, Bessel functions, modified Bessel functions, Dunkl kernels

2010 Mathematics Subject Classification: 26D07, 33B10, 33C10.

Cite This Article: Frej Chouchene, "Oppenheim's Problem and Some Inequalities Involving Bessel Functions and Dunkl Kernels." Turkish Journal of Analysis and Number Theory, vol. 6, no. 3 (2018): 61-71. doi: 10.12691/tjant-6-3-1.

\section{Introduction}

We consider the Oppenheim's problem: What are the best possible constants $l_{1}, l_{2}, r_{1}, r_{2} \in \mathbb{R}$ such that

$$
l_{1} \cos x+l_{2} \leq \frac{\sin x}{x} \leq r_{1} \cos x+r_{2}
$$

hold for all $x \in[-\pi, \pi] \backslash\{0\}$ ?

In 1958, a partial solution is given in $[1,2]$ : If $\left.a \in] 0, \frac{1}{2}\right]$ and $x \in\left[-\frac{\pi}{2}, \frac{\pi}{2}\right]$, then

$$
\frac{(a+1) \sin x}{1+a \cos x} \leq x \leq \frac{\pi}{2} \frac{\sin x}{1+a \cos x} .
$$

Next, L. Zhu solved completely this problem for trigonometric functions, see [3]. Since the cosine and sine functions are particular cases of Bessel functions, then it is natural to generalize some formulas and inequalities involving these elementary functions to Bessel functions. The extension of the Oppenheim's problem to Bessel and modified Bessel functions was first considered by Á. Baricz in [4]. Recently, we established in [5] some inequalities related to this type of problem for Dunkl kernels $\psi_{-i}^{\alpha}$ by answering to the following question: What are, for $\alpha \geq-\frac{1}{2}$, the best possible constants $l_{1}, l_{2}, r_{1}, r_{2} \in \mathbb{R}$ such that

$$
l_{1} \psi_{-i}^{\alpha}(x)+l_{2} \leq \psi_{-i}^{\alpha+1}(x) \leq r_{1} \psi_{-i}^{\alpha}(x)+r_{2}
$$

hold for all $x \in I ; \quad I=\mathbb{R},[a, b], a, b \in \mathbb{R}$;

$$
a<b,[a,+\infty[, a \in \mathbb{R},]-\infty, b], b \in \mathbb{R} . ?
$$

Some new inequalities involving modified Bessel functions have also been improved.

Our aim is to solve the analogues of the Oppenheim's problem for the real and imaginary parts of Dunkl kernels $\psi_{1}^{\alpha}, \alpha \geq-\frac{1}{2}$. In the beginning, we present some new inequalities related to this problem for trigonometric functions. These inequalities and Sonine integral formula for Bessel functions allow us to get a new version of the solution of this type of problem for Bessel functions $\mathcal{J}_{\alpha}, \alpha \geq-\frac{1}{2}$. Next, by using again Sonine integral formula for Bessel functions, we solve the Oppenheim's problem for the imaginary parts of Dunkl kernels $\psi_{1}^{\alpha}, \alpha \geq-\frac{1}{2}$. At the end of this paper, we give refinements of inequalities for Bessel functions $\mathcal{J}_{\alpha}, \alpha \geq-\frac{1}{2}$. More precisely, in view of the inequalities given by Á. Baricz in $[4,6]$, we prove that if $\left.\alpha \geq-\frac{1}{2}, a_{1} \in\right] 0, \frac{1}{2}\left[\right.$ and $\left.a_{2}:=a_{1}+1 \in\right] 1, \frac{3}{2}[$, then for all $x \in[-\pi, \pi] \backslash\{0\}$, we have

$$
\frac{(\alpha+1) \mathcal{J}_{\alpha}(x)+1}{\alpha+2}<\frac{2(\alpha+1) a_{1} \mathcal{J}_{\alpha}(x)+1}{(2 \alpha+1) a_{1}+a_{2}}
$$

and 


$$
\frac{(\alpha+1) \mathcal{J}_{\alpha}(0)+1}{\alpha+2}=\frac{2(\alpha+1) a_{1} \mathcal{J}_{\alpha}(0)+1}{(2 \alpha+1) a_{1}+a_{2}}=1 .
$$

If $\left.\alpha \geq-\frac{1}{2}, a_{1} \in\right] 0,+\infty\left[\backslash\left\{\frac{\pi-2}{2}\right\}, \quad\right.$ then for all $x \in[-\pi, \pi] \backslash\left\{-\frac{\pi}{2}, 0, \frac{\pi}{2}\right\}$, we have

$$
\frac{2(\alpha+1) a_{1} \mathcal{J}_{\alpha}(x)+1}{(2 \alpha+1) a_{1}+a_{3}}<\frac{(\alpha+1)(\pi-2) \mathcal{J}_{\alpha}(x)+1}{(\alpha+1) \pi-(2 \alpha+1)},
$$

where $a_{1}, a_{2}$ and $a_{3}$ are as in Theorem 1.1 of [6].

\section{Dunkl Kernels}

In this section, we take $\alpha>-1$.

Definition 2.1. Let $\lambda \in \mathbb{C}$. We call Dunkl kernel the function $\psi_{\lambda}^{\alpha}$ defined by

$$
\psi_{\lambda}^{\alpha}(z):=\mathcal{J}_{\alpha}(\lambda z)+\frac{i \lambda z}{2(\alpha+1)} \mathcal{J}_{\alpha+1}(\lambda z), z \in \mathbb{C},
$$

where $\mathcal{J}_{\alpha}$ is the normalized Bessel function of index $\alpha$ given by

$$
\mathcal{J}_{\alpha}(z):=\sum_{n=0}^{+\infty}(-1)^{n} c_{n}(\alpha) z^{2 n}
$$

with for all $n \in \mathbb{N}$, we have

$$
c_{n}(\alpha):=\frac{\Gamma(\alpha+1)}{2^{2 n} n ! \Gamma(\alpha+n+1)} .
$$

For more details of these functions, we can see [7-17]. The following results of this section are proved in [5].

Proposition 2.2. For all $\lambda, z \in \mathbb{C}, x \in \mathbb{R}$ and $n \in \mathbb{N}$, we have

1) $\psi_{\lambda}^{\alpha}(z)=\psi_{z}^{\alpha}(\lambda)=\psi_{\lambda z}^{\alpha}(1)$.

2) $\psi_{-\lambda}^{\alpha}(z)=\psi_{\lambda}^{\alpha}(-z), \psi_{-\lambda}^{\alpha}(-z)=\psi_{\lambda}^{\alpha}(z)$,

$$
\overline{\psi_{\lambda}^{\alpha}(z)}=\psi \frac{\alpha}{\lambda}(-\bar{z})
$$

3) $\psi_{\lambda}^{\alpha}(0)=1$.

4)

$$
\psi_{\lambda}^{\alpha}(z)=\Gamma(\alpha+1)\left(\begin{array}{l}
\sum_{n=0}^{+\infty} \frac{(-1)^{n}(\lambda z)^{2 n}}{2^{2 n} n ! \Gamma(\alpha+n+1)} \\
+i \sum_{n=0}^{+\infty} \frac{(-1)^{n}(\lambda z)^{2 n+1}}{2^{2 n+1} n ! \Gamma(\alpha+n+2)}
\end{array}\right) .
$$

5)

$$
\begin{aligned}
\psi_{\lambda}^{\alpha}(z) & =\frac{\Gamma(\alpha+1)}{\sqrt{\pi} \Gamma\left(\alpha+\frac{1}{2}\right)} \\
& \times \int_{-1}^{1}(1-t)^{\alpha+\frac{1}{2}}(1+t)^{\alpha-\frac{1}{2}} e^{-i \lambda z t} d t
\end{aligned}
$$

6) $\left|\psi_{\lambda}^{\alpha}(z)\right| \leq e^{|\Im(\lambda z)|}$.
7) $\left|\frac{d^{n}}{d x^{n}}\left(\psi_{\lambda}^{\alpha}\right)(x)\right| \leq|\lambda|^{n} \psi_{i \Im \lambda}^{\alpha}(x)$.

In particular, if $\lambda \in \mathbb{R}$ then $\left|\psi_{\lambda}^{\alpha}(x)\right| \leq 1$.

8) $\lim _{\lambda \rightarrow \pm \infty} \psi_{\lambda}^{\alpha}(x)=0, \quad x \neq 0$.

Proposition 2.3. The Dunkl kernel $\psi_{\lambda}^{\alpha}, \lambda \in \mathbb{C}$, is the unique entire solution on $\mathbb{R}$ of the following equation:

$$
\left\{\begin{array}{l}
\Lambda_{\alpha} u=i \lambda u \\
u(0)=1
\end{array}\right.
$$

where $\Lambda_{\alpha}$ is the Dunkl operator on $\mathbb{R}$ of index $\left(\alpha+\frac{1}{2}\right)$ associated to the reflections group $\mathbb{Z}_{2}$ and given by

$$
\Lambda_{\alpha} f(x):=\frac{d}{d x} f(x)+\frac{2 \alpha+1}{x}\left(\frac{f(x)-f(-x)}{2}\right),
$$

$f \in C^{1}(\mathbb{R}), \alpha>-\frac{1}{2}$.

Remarks 2.4. If $f \in C^{1}(\mathbb{R})$, then we have

1) $\Lambda_{\alpha} f(0)=2(\alpha+1) \frac{d}{d x} f(0)$.

2) $\left(\Lambda_{\alpha} f\right)_{e}=\Lambda_{\alpha}\left(f_{o}\right)=\frac{d}{d x} f_{o}+(2 \alpha+1) \frac{f_{o}}{x}$.

3) $\left(\Lambda_{\alpha} f\right)_{o}=\Lambda_{\alpha}\left(f_{e}\right)=\frac{d}{d x} f_{e}$.

4)

$$
\Lambda_{\alpha} f(x)=\frac{d}{d y} f(x)+\left(\alpha+\frac{1}{2}\right) \int_{-1}^{1} \frac{d}{d y} f(x t) d t, x \in \mathbb{R}
$$

where $f_{e}$ (resp. $f_{o}$ ) is the even (resp. odd) part of $f$.

In particular, for all $z \in \mathbb{C}$, we have

$$
\psi_{1}^{\alpha}(z)=\mathcal{J}_{\alpha}(z)+i \frac{z}{2(\alpha+1)} \mathcal{J}_{\alpha+1}(z),
$$

where $\mathcal{J}_{\alpha}$ is the normalized Bessel function of index $\alpha$.

Proposition 2.5. For all $z \in \mathbb{C}, x \in \mathbb{R}$ and $n \in \mathbb{N}$, we have

1) $\psi_{1}^{\alpha}(0)=1$

2) $\psi_{1}^{\alpha}(z)=\sum_{k=0}^{+\infty} b_{k}(\alpha) z^{k}$, with for all $k \in \mathbb{N}$, we have

$$
\left\{\begin{aligned}
b_{2 k}(\alpha) & :=(-1)^{k} c_{k}(\alpha) \\
b_{2 k+1}(\alpha) & :=i \frac{(-1)^{k} c_{k}(\alpha)}{2(k+\alpha+1)}
\end{aligned}\right.
$$

where $c_{k}(\alpha)$ is given by (1).

3)

$$
\psi_{1}^{\alpha}(z)=\frac{\Gamma(\alpha+1)}{\sqrt{\pi} \Gamma\left(\alpha+\frac{1}{2}\right)} \times \int_{-1}^{1}(1-t)^{\alpha+\frac{1}{2}}(1+t)^{\alpha-\frac{1}{2}} e^{-i z t} d t
$$

4) $\left|\psi_{1}^{\alpha}(z)\right| \leq e^{|\mathfrak{\Im} z|}$. 
5)

$\frac{d^{n}}{d x^{n}}\left(\psi_{1}^{\alpha}\right)(x)=\sum_{k=0}^{+\infty} \frac{(n+k) !}{k !} b_{n+k}(\alpha) x^{k}$

$=\frac{\Gamma(\alpha+1)}{\sqrt{\pi} \Gamma\left(\alpha+\frac{1}{2}\right)} \int_{-1}^{1}(-i t)^{n}(1-t)^{\alpha+\frac{1}{2}}(1+t)^{\alpha-\frac{1}{2}} e^{-i x t} d t$.

6) $\left|\frac{d^{n}}{d x^{n}}\left(\psi_{1}^{\alpha}\right)(x)\right| \leq 1$.

7) The function $\psi_{1}^{\alpha}$ is the unique entire solution on $\mathbb{R}$ of the following equation:

$$
\left\{\begin{array}{l}
\Lambda_{\alpha} u=i u \\
u(0)=1
\end{array}\right.
$$

Examples 2.6. For all $x \in \mathbb{R} \backslash\{0\}$, we have

$$
\begin{aligned}
& \text { 1) } \mathcal{J}_{-\frac{1}{2}}(x)=\cos x, \\
& \mathcal{I}_{-\frac{1}{2}}(x)=\cosh x, \\
& \psi_{1}^{-\frac{1}{2}}(x)=e^{i x}, \quad x \in \mathbb{R} . \\
& \text { 2) } \mathcal{J}_{\frac{1}{2}}(x)=\frac{\sin x}{x}, \\
& \mathcal{I}_{\frac{1}{2}}(x)=\frac{\sinh x}{x}, \\
& \psi_{1}^{\frac{1}{2}}(x)=\frac{x \sin x+i(\sin x-x \cos x)}{x^{2}} . \\
& \mathcal{J}_{\frac{3}{2}}(x)=3 \frac{\sin x-x \cos x}{x^{3}}, \\
& \mathcal{I}_{\frac{3}{2}}(x)=3 \frac{x \cosh x-\sinh x}{x^{3}}, \\
& \psi_{1}^{\frac{3}{2}}(x)=\frac{x(\sin x-x \cos x)-i\left[3 x \cos x+\left(x^{2}-3\right) \sin x\right]}{x^{4}}
\end{aligned}
$$

where $\mathcal{I}_{\alpha}$ is the normalized modified Bessel function of index $\alpha$ given by

$$
\mathcal{I}_{\alpha}(z):=\sum_{n=0}^{+\infty} c_{n}(\alpha) z^{2 n},
$$

with $c_{n}(\alpha)$ is given by (1).

\section{Remarks 2.7.}

1) The function $\mathbf{J}_{\alpha}$ is the unique entire solution on $\mathbb{R}$ of the following equation:

$$
\left\{\begin{aligned}
L_{\alpha} u & =-u, \\
u(0) & =1, \\
\frac{d}{d x} u(0) & =0 .
\end{aligned}\right.
$$

2) The function $\mathcal{I}_{\alpha}$ is the unique entire solution on $\mathbb{R}$ of the following equation:

$$
\left\{\begin{aligned}
L_{\alpha} u & =u, \\
u(0) & =1, \\
\frac{d}{d x} u(0) & =0,
\end{aligned}\right.
$$

where $L_{\alpha}$ is the Bessel operator on $\mathbb{R}$ given by

$$
L_{\alpha} f(x):=\frac{d^{2}}{d x^{2}} f(x)+\frac{2 \alpha+1}{x} \frac{d}{d x} f(x),
$$

$f \in C^{2}(\mathbb{R}), \alpha>-\frac{1}{2}$.

Proposition 2.8. For all $\alpha>0$ and $x \in \mathbb{R}$, we have

1) $\frac{d}{d x} \mathcal{J}_{\alpha}(x)=-\frac{x}{2(\alpha+1)} \mathcal{J}_{\alpha+1}(x)$.

2) $\mathcal{J}_{\alpha-1}(x)=\mathcal{J}_{\alpha}(x)-\frac{x^{2}}{4 \alpha(\alpha+1)} \mathcal{J}_{\alpha+1}(x)$.

3) $\frac{d}{d x} \mathcal{I}_{\alpha}(x)=\frac{x}{2(\alpha+1)} \mathcal{I}_{\alpha+1}(x)$.

4) $\mathcal{I}_{\alpha-1}(x)=\mathcal{I}_{\alpha}(x)+\frac{x^{2}}{4 \alpha(\alpha+1)} \mathcal{I}_{\alpha+1}(x)$.

Proposition 2.9. For all $k, n \in \mathbb{N}$ and $x \in \mathbb{R}$, we have
1) $\frac{d^{2 k}}{d x^{2 k}}\left[\psi_{1}^{\alpha}(-i x)\right]>0$.
2) If $x \geq-1$, then $\frac{d^{2 k+1}}{d x^{2 k+1}}\left[\psi_{1}^{\alpha}(-i x)\right]>0$.
3) If $x \geq-1$, then $\frac{d^{n}}{d x^{n}}\left[\psi_{1}^{\alpha}(-i x)\right]>0$.

\section{Inequalities Related to Oppenheim's Problem}

\subsection{Main Results}

We begin to find the best possible constants $l_{1}, l_{2}, r_{1}, r_{2} \in \mathbb{R}$ such that

$$
l_{1} \mathcal{J}_{-\frac{1}{2}}(x)+l_{2} \leq \mathcal{J}_{\frac{1}{2}}(x) \leq r_{1} \mathcal{J}_{-\frac{1}{2}}(x)+r_{2}
$$

hold for all $x \in I ; \quad I=[-\pi, \pi],[-b, b]$,

$$
0<b<\pi,[-\pi,-a] \cup[a, \pi], 0<a<\pi .
$$

The solution of this problem can be stated in the following theorem:

Theorem 3.1

1) For all $b \in] 0, \pi[$ and $x \in[-\pi, \pi]$, we have

$$
\begin{aligned}
& \sup \left\{\mathcal{J}_{-\frac{1}{2}}(x), 0\right\} \leq \frac{1}{2}\left(\mathcal{J}_{-\frac{1}{2}}(x)+1\right) \leq \mathcal{J}_{\frac{1}{2}}(x) \\
\leq & \inf \left\{\frac{1}{3}\left(\mathcal{J}_{-\frac{1}{2}}(x)+2\right), u_{1}(b) \mathcal{J}_{-\frac{1}{2}}(x)+u_{4}(b)\right\} .
\end{aligned}
$$


2) For all $\left.b \in] 0, \frac{\pi}{2}\right]$ and $x \in[-b, b]$, we have

$$
\begin{aligned}
& \left(1-u_{4}(b)\right) \mathcal{J}_{-\frac{1}{2}}(x)+u_{4}(b) \leq \mathcal{J}_{\frac{1}{2}}(x) \\
& \leq \inf \left\{\frac{1}{3}\left(\mathcal{J}_{-\frac{1}{2}}(x)+2\right), u_{1}(b) \mathcal{J}_{-\frac{1}{2}}(x)+u_{4}(b)\right\} .
\end{aligned}
$$

3) For all $b \in] \frac{\pi}{2}, \pi[$ and $x \in[-b, b]$, we have

$$
\begin{aligned}
& \frac{1}{1-w_{1}(b)}\left(\mathcal{J}_{-\frac{1}{2}}(x)-w_{1}(b)\right) \leq \mathcal{J}_{\frac{1}{2}}(x) \\
& \leq \inf \left\{\frac{1}{3}\left(\mathcal{J}_{-\frac{1}{2}}(x)+2\right), u_{1}(b) \mathcal{J}_{-\frac{1}{2}}(x)+u_{4}(b)\right\} .
\end{aligned}
$$

4) For all $a \in\left[0, \frac{\pi}{2}[\right.$ and $x \in[-\pi,-a] \cup[a, \pi]$, we have

$$
\begin{aligned}
& u_{1}(a)\left(\mathcal{J}_{-\frac{1}{2}}(x)+1\right) \leq \mathcal{J}_{\frac{1}{2}}(x) \\
& \leq u_{1}(a) \mathcal{J}_{-\frac{1}{2}}(x)+u_{4}(a) .
\end{aligned}
$$

5) For all $a \in\left[\frac{\pi}{2}, \pi[\right.$ and $x \in[-\pi,-a] \cup[a, \pi]$, we have

$$
\begin{aligned}
& u_{4}(a)\left(\mathcal{J}_{-\frac{1}{2}}(x)+1\right) \leq \mathcal{J}_{\frac{1}{2}}(x) \\
& \leq u_{1}(a) \mathcal{J}_{-\frac{1}{2}}(x)+u_{4}(a),
\end{aligned}
$$

where $u_{1}, u_{4}$ and $w_{1}$ are respectively given by (4), (5) and (6).

With the aid of Sonine integral formula for Bessel functions we get the following theorem:

Theorem 3.2 Let $\alpha \geq-\frac{1}{2}$.

1) For all $b \in] 0, \pi[$ and $x \in[-\pi, \pi]$, we have

$$
\begin{aligned}
& \frac{2(\alpha+1) \mathcal{J}_{\alpha}(x)+1}{2 \alpha+3} \leq \mathcal{J}_{\alpha+1}(x) \leq \\
& \inf \left\{\frac{(\alpha+1) \mathcal{J}_{\alpha}(x)+1}{\alpha+2}, \frac{2(\alpha+1) u_{1}(b) \mathcal{J}_{\alpha}(x)+u_{4}(b)}{(2 \alpha+1) u_{1}(b)+1}\right\} .
\end{aligned}
$$

2) For all $\left.b \in] 0, \frac{\pi}{2}\right]$ and $x \in[-b, b]$, we have

$$
\begin{aligned}
& \frac{2(\alpha+1)\left(1-u_{4}(b)\right) \mathcal{J}_{\alpha}(x)+u_{4}(b)}{2(\alpha+1)-(2 \alpha+1) u_{4}(b)} \leq \mathcal{J}_{\alpha+1}(x) \\
& \leq \inf \left\{\frac{(\alpha+1) \mathcal{J}_{\alpha}(x)+1}{\alpha+2}, \frac{2(\alpha+1) u_{1}(b) \mathcal{J}_{\alpha}(x)+u_{4}(b)}{(2 \alpha+1) u_{1}(b)+1}\right\} .
\end{aligned}
$$

3) For all $b \in] \frac{\pi}{2}, \pi[$ and $x \in[-b, b]$, we have

$$
\begin{aligned}
& \frac{2(\alpha+1) \mathcal{J}_{\alpha}(x)-w_{1}(b)}{2(\alpha+1)-w_{1}(b)} \leq \mathcal{J}_{\alpha+1}(x) \\
& \leq \inf \left\{\frac{(\alpha+1) \mathcal{J}_{\alpha}(x)+1}{\alpha+2},\right. \\
& \left.\frac{2(\alpha+1) u_{1}(b) \mathcal{J}_{\alpha}(x)+u_{4}(b)}{(2 \alpha+1) u_{1}(b)+1}\right\},
\end{aligned}
$$

where $u_{1}, u_{4}$ and $w_{1}$ are respectively given by (4), (5) and (6).

Thus, by choosing $\alpha=\frac{1}{2}$ in Theorem 3.2, we obtain the following interesting result.

\section{Corollary 3.3}

1) For all $b \in] 0, \pi[$ and $x \in[-\pi, \pi]$, we have

$$
\begin{aligned}
& \sup \left\{\frac{15(\sin x-x \cos x)}{3 \sin x+2 x},\right. \\
& \left.\frac{3\left(2 u_{1}(b)+1\right)(\sin x-x \cos x)}{3 u_{1}(b) \sin x+u_{4}(b) x}\right\} \\
& \leq x^{2} \leq \frac{12(\sin x-x \cos x)}{3 \sin x+x} .
\end{aligned}
$$

2) For all $x \in[-\pi, \pi]$, we have

$$
\begin{aligned}
& \sup \left\{\frac{15(\sin x-x \cos x)}{3 \sin x+2 x}, \frac{3\left(\pi^{2}+8\right)(\sin x-x \cos x)}{2(6 \sin x+\pi x)}\right\} \\
& \leq x^{2} \leq \frac{12(\sin x-x \cos x)}{3 \sin x+x}
\end{aligned}
$$

3) For all $\left.b \in] 0, \frac{\pi}{2}\right]$ and $x \in[-b, b]$, we have $\sup \left\{\frac{15(\sin x-x \cos x)}{3 \sin x+2 x}, \frac{3\left(2 u_{1}(b)+1\right)(\sin x-x \cos x)}{3 u_{1}(b) \sin x+u_{4}(b) x}\right\}$ $\leq x^{2} \leq \frac{3\left(3-2 u_{4}(b)\right)(\sin x-x \cos x)}{3\left(1-u_{4}(b)\right) \sin x+u_{4}(b) x}$.

4) For all $x \in\left[-\frac{\pi}{2}, \frac{\pi}{2}\right]$, we have

$$
\begin{aligned}
& \sup \left\{\frac{15(\sin x-x \cos x)}{3 \sin x+2 x}, \frac{3\left(\pi^{2}+8\right)(\sin x-x \cos x)}{2(6 \sin x+\pi x)}\right\} \\
& \leq x^{2} \leq \frac{3(3 \pi-4)(\sin x-x \cos x)}{3(\pi-2) \sin x+2 x}
\end{aligned}
$$

5) For all $b \in] \frac{\pi}{2}, \pi[$ and $x \in[-b, b]$, we have

$$
\begin{aligned}
& \sup \left\{\frac{15(\sin x-x \cos x)}{3 \sin x+2 x},\right. \\
& \left.\frac{3\left(2 u_{1}(b)+1\right)(\sin x-x \cos x)}{3 u_{1}(b) \sin x+u_{4}(b) x}\right\} \\
& \leq x^{2} \leq \frac{3\left(3-w_{1}(b)\right)(\sin x-x \cos x)}{3 \sin x-w_{1}(b) x},
\end{aligned}
$$


where $u_{1}, u_{4}$ and $w_{1}$ are respectively given by (4), (5) and (6).

Now, for $\alpha \geq-\frac{1}{2}$, we are going to find the best possible constants $l_{1}, l_{2}, r_{1}, r_{2} \in \mathbb{R}$ such that

$$
l_{1} \Im \psi_{1}^{\alpha}(x)+l_{2} \leq \Im \psi_{1}^{\alpha+1}(x) \leq r_{1} \Im \psi_{1}^{\alpha}(x)+r_{2}
$$

hold for all $x \in I ; \quad I=[0, \pi], \quad[-\pi, 0], \quad[0, b], \quad[-b, 0]$, $0<b<\pi$.

The solution of this problem can be stated in the following theorem:

Theorem 3.4 Let $\alpha \geq-\frac{1}{2}$.

1) For all $b \in] 0, \pi[$ and $x \in[0, \pi]$, we have

$$
\begin{aligned}
& \frac{6(\alpha+1) \mathfrak{I} \psi_{1}^{\alpha}(x)+x}{3(2 \alpha+5)} \leq \mathfrak{I} \psi_{1}^{\alpha+1}(x) \\
& \leq \inf \left\{\frac{3(\alpha+1) \mathfrak{I} \psi_{1}^{\alpha}(x)+x}{3(\alpha+3)},\right. \\
& \left.\frac{6(\alpha+1) u_{1}(b) \mathfrak{I} \psi_{1}^{\alpha}(x)+u_{4}(b) x}{3\left[(2 \alpha+3) u_{1}(b)+1\right]}\right\} .
\end{aligned}
$$

2) For all $b \in] 0, \pi[$ and $x \in[-\pi, 0]$, we have

$$
\begin{aligned}
& \inf \left\{\frac{3(\alpha+1) \mathfrak{I} \psi_{1}^{\alpha}(x)+x}{3(\alpha+3)},\right. \\
& \left.\frac{6(\alpha+1) u_{1}(b) \Im \psi_{1}^{\alpha}(x)+u_{4}(b) x}{3\left[(2 \alpha+3) u_{1}(b)+1\right]}\right\} \\
& \leq \mathfrak{I} \psi_{1}^{\alpha+1}(x) \leq \frac{6(\alpha+1) \mathfrak{I} \psi_{1}^{\alpha}(x)+x}{3(2 \alpha+5)} .
\end{aligned}
$$

3) For all $\left.b \in] 0, \frac{\pi}{2}\right]$ and $x \in[0, b]$, we have

$$
\begin{aligned}
& \frac{6(\alpha+1)\left(1-u_{4}(b)\right) \mathfrak{I} \psi_{1}^{\alpha}(x)+u_{4}(b) x}{3\left[2(\alpha+2)-(2 \alpha+3) u_{4}(b)\right]} \\
& \leq \mathfrak{I} \psi_{1}^{\alpha+1}(x) \leq \inf \left\{\frac{3(\alpha+1) \mathfrak{I} \psi_{1}^{\alpha}(x)+x}{3(\alpha+3)},\right. \\
& \left.\frac{6(\alpha+1) u_{1}(b) \Im \psi_{1}^{\alpha}(x)+u_{4}(b) x}{3\left[(2 \alpha+3) u_{1}(b)+1\right]}\right\} .
\end{aligned}
$$

4) For all $\left.b \in] 0, \frac{\pi}{2}\right]$ and $x \in[-b, 0]$, we have

$$
\begin{aligned}
& \inf \left\{\frac{3(\alpha+1) \Im \psi_{1}^{\alpha}(x)+x}{3(\alpha+3)},\right. \\
& \left.\frac{6(\alpha+1) u_{1}(b) \Im \psi_{1}^{\alpha}(x)+u_{4}(b) x}{3\left[(2 \alpha+3) u_{1}(b)+1\right]}\right\} \\
& \leq \Im \psi_{1}^{\alpha+1}(x) \\
& \leq \frac{6(\alpha+1)\left(1-u_{4}(b)\right) \Im \psi_{1}^{\alpha}(x)+u_{4}(b) x}{3\left[2(\alpha+2)-(2 \alpha+3) u_{4}(b)\right]} .
\end{aligned}
$$

5) For all $b \in] \frac{\pi}{2}, \pi[$ and $x \in[0, b]$, we have

$$
\begin{aligned}
& \frac{6(\alpha+1) \mathfrak{I} \psi_{1}^{\alpha}(x)-w_{1}(b) x}{3\left[2(\alpha+2)-w_{1}(b)\right]} \\
& \leq \mathfrak{I} \psi_{1}^{\alpha+1}(x) \leq \inf \left\{\frac{3(\alpha+1) \mathfrak{I} \psi_{1}^{\alpha}(x)+x}{3(\alpha+3)},\right. \\
& \left.\frac{6(\alpha+1) u_{1}(b) \Im \psi_{1}^{\alpha}(x)+u_{4}(b) x}{3\left[(2 \alpha+3) u_{1}(b)+1\right]}\right\} .
\end{aligned}
$$

6) For all $b \in] \frac{\pi}{2}, \pi[$ and $x \in[-b, 0]$, we have

$$
\begin{aligned}
& \inf \left\{\frac{3(\alpha+1) \mathfrak{I} \psi_{1}^{\alpha}(x)+x}{3(\alpha+3)},\right. \\
& \left.\frac{6(\alpha+1) u_{1}(b) \Im \psi_{1}^{\alpha}(x)+u_{4}(b) x}{3\left[(2 \alpha+3) u_{1}(b)+1\right]}\right\} \\
& \leq \mathfrak{I} \psi_{1}^{\alpha+1}(x) \leq \frac{6(\alpha+1) \mathfrak{I} \psi_{1}^{\alpha}(x)-w_{1}(b) x}{3\left[2(\alpha+2)-w_{1}(b)\right]},
\end{aligned}
$$

where $u_{1}, u_{4}$ and $w_{1}$ are respectively given by (4), (5) and (6).

\subsection{Preliminary Results}

In order to solve Problem (3), we present the following propositions:

\section{Proposition 3.5}

1) For all $b \in] 0, \pi[$ and $x \in[-\pi, \pi]$, we have

$$
\begin{aligned}
& u_{1}(b) \mathcal{J}_{-\frac{1}{2}}(x)+\inf \left\{1-u_{1}(b), u_{1}(b)\right\} \\
& \leq \mathcal{J}_{\frac{1}{2}}(x) \leq u_{1}(b) \mathcal{J}_{-\frac{1}{2}}(x)+u_{4}(b) .
\end{aligned}
$$

2) For all $b \in] 0, \pi[$ and $x \in[-b, b]$, we have

$$
\begin{aligned}
& u_{1}(b) \mathcal{J}_{-\frac{1}{2}}(x)+1-u_{1}(b) \\
& \leq \mathcal{J}_{\frac{1}{2}}(x) \leq u_{1}(b) \mathcal{J}_{-\frac{1}{2}}(x)+u_{4}(b) .
\end{aligned}
$$

3) For all $a \in[0, \pi[$ and $x \in[-\pi,-a] \cup[a, \pi]$, we have

$$
u_{1}(a)\left(\mathcal{J}_{-\frac{1}{2}}(x)+1\right) \leq \mathcal{J}_{\frac{1}{2}}(x) \leq u_{1}(a) \mathcal{J}_{-\frac{1}{2}}(x)+u_{4}(a),
$$

where for $x \in]-\pi, \pi[\backslash\{0\}$,

$$
\begin{gathered}
u_{1}(x):=\frac{\frac{\mathcal{J}_{3}}{2}(x)}{3 \mathcal{J}_{\frac{1}{2}}(x)}=\frac{\sin x-x \cos x}{x^{2} \sin x}, \\
u_{4}(x):=\mathcal{J}_{\frac{1}{2}}(x)-\cos x u_{1}(x)=\frac{2 x-\sin (2 x)}{2 x^{2} \sin x} .
\end{gathered}
$$




\section{Proposition 3.6}

1) For all $\left.b \in] 0, \frac{\pi}{2}\right]$ and $x \in[-b, b]$, we have

$$
\begin{aligned}
& \left(1-u_{4}(b)\right) \mathcal{J}_{-\frac{1}{2}}(x)+u_{4}(b) \\
& \leq \mathcal{J}_{\frac{1}{2}}(x) \leq u_{1}(b) \mathcal{J}_{-\frac{1}{2}}(x)+u_{4}(b) .
\end{aligned}
$$

2) For all $a \in\left[0, \frac{\pi}{2}\left[\right.\right.$ and $x \in\left[-\frac{\pi}{2},-a\right] \cup\left[a, \frac{\pi}{2}\right]$, we have

$$
\mathcal{J}_{\frac{1}{2}}(x) \leq u_{1}(a) \mathcal{J}_{-\frac{1}{2}}(x)+u_{4}(a) .
$$

3) For all $b \in] \frac{\pi}{2}, \pi\left[\right.$ and $x \in\left[-b,-\frac{\pi}{2}\right] \cup\left[\frac{\pi}{2}, b\right]$, we have

$$
\mathcal{J}_{\frac{1}{2}}(x) \leq u_{1}(b) \mathcal{J}_{-\frac{1}{2}}(x)+u_{4}(b) .
$$

4) For all $a \in\left[\frac{\pi}{2}, \pi[\right.$ and $x \in[-\pi,-a] \cup[a, \pi]$, we have

$$
\begin{aligned}
& u_{4}(a)\left(\mathcal{J}_{-\frac{1}{2}}(x)+1\right) \leq \mathcal{J}_{\frac{1}{2}}(x) \\
& \leq u_{1}(a) \mathcal{J}_{-\frac{1}{2}}(x)+u_{4}(a),
\end{aligned}
$$

where $u_{1}$ and $u_{4}$ are respectively given by (4) and (5).

\section{Proposition 3.7}

1) For all $x \in[-\pi, \pi]$, we have

$$
\begin{aligned}
& \sup \left\{\mathcal{J}_{-\frac{1}{2}}(x), 0\right\} \leq \frac{1}{2}\left(\mathcal{J}_{-\frac{1}{2}}(x)+1\right) \\
& \leq \mathcal{J}_{\frac{1}{2}}(x) \leq \frac{1}{3}\left(\mathcal{J}_{-\frac{1}{2}}(x)+2\right) \leq 1 .
\end{aligned}
$$

2) For all $b \in] 0, \pi[$ and $x \in[-\pi, \pi]$, we have

$$
\mathcal{J}_{\frac{1}{2}}(x) \leq u_{1}(b) \mathcal{J}_{-\frac{1}{2}}(x)+u_{4}(b) .
$$

3) For all $a \in] 0, \pi[$ and $x \in[-\pi,-a] \cup[a, \pi]$, we have

$$
\mathcal{J}_{\frac{1}{2}}(x) \leq u_{1}(a) \mathcal{J}_{-\frac{1}{2}}(x)+u_{4}(a) .
$$

4) For all $b \in] 0, \pi[$ and $x \in[-b, b]$, we have

$$
\begin{aligned}
& \frac{1}{1-w_{1}(b)}\left(\mathcal{J}_{-\frac{1}{2}}(x)-w_{1}(b)\right) \\
& \leq \mathcal{J}_{\frac{1}{2}}(x) \leq u_{1}(b) \mathcal{J}_{-\frac{1}{2}}(x)+u_{4}(b),
\end{aligned}
$$

where $u_{1}, u_{4}$ and $w_{1}$ are respectively given by (4), (5) and for $x \in[-\pi, \pi] \backslash\{0\}$,

$$
\begin{aligned}
& w_{1}(x):=-\frac{u_{4}(x)}{u_{1}(x)}=-3 \frac{1-\mathcal{J}_{\frac{1}{2}}(2 x)}{x^{2} \mathcal{J}_{\frac{3}{2}}(x)} \\
& =-\frac{2 x-\sin (2 x)}{2(\sin x-x \cos x)} .
\end{aligned}
$$

The study of the Bessel function $\mathcal{J}_{3}$ gives the following proposition:

Proposition 3.8 The function $\mathcal{J}_{\frac{3}{2}}$ is even on $\mathbb{R}$, strictly increasing on $[-\pi, 0]$, strictly decreasing on $[0, \pi]$, and satisfies

1) $\mathcal{J}_{\frac{3}{2}}(0)=1, \mathcal{J}_{\frac{3}{2}}\left( \pm \frac{\pi}{2}\right)=\frac{24}{\pi^{3}}, \mathcal{J}_{\frac{3}{2}}( \pm \pi)=\frac{3}{\pi^{2}}$.

2) $\forall x \in \mathbb{R}, \frac{x^{2}}{3} \mathcal{J}_{\frac{3}{2}}(x)=\mathcal{J}_{\frac{1}{2}}(x)-\mathcal{J}_{-\frac{1}{2}}(x)$.

3) $\forall x \in[-\pi, \pi], \mathcal{J}_{\frac{1}{2}}(x) \leq \mathcal{J}_{-\frac{1}{2}}(x)+\frac{x^{2}}{3}$.

In view of [18] we deduce the following Sonine integral formulas:

Proposition 3.9 For all $\alpha>\beta>-1$ and $x \in \mathbb{R}$, we have

$\mathcal{J}_{\alpha}(x)=\frac{2}{B(\alpha-\beta, \beta+1)} \int_{0}^{1} \mathcal{J}_{\beta}(x t) t^{2 \beta+1}\left(1-t^{2}\right)^{\alpha-\beta-1} d t$ $=\frac{2}{B(\alpha-\beta, \beta+1)} \int_{0}^{\frac{\pi}{2}} \mathcal{J}_{\beta}(x \sin \theta)(\sin \theta)^{2 \beta+1}(\cos \theta)^{2(\alpha-\beta)-1} d \theta$.

2)

$$
\psi_{1}^{\alpha}(x)=\frac{1}{B(\alpha-\beta, \beta+1)}
$$$$
\times \int_{-1}^{1} \psi_{1}^{\beta}(x t)|t|^{2 \beta+1}(1+t)\left(1-t^{2}\right)^{\alpha-\beta-1} d t
$$$$
=\frac{1}{B(\alpha-\beta, \beta+1)}
$$

$\times \int_{-\frac{\pi}{2}}^{\frac{\pi}{2}} \psi_{1}^{\beta}(x \sin \theta)(1+\sin \theta)(\sin (|\theta|))^{2 \beta+1}(\cos \theta)^{2(\alpha-\beta)-1} d \theta$

$$
=\frac{2}{B(\alpha-\beta, \beta+1)}
$$

$\times \int_{0}^{1}\left[\mathcal{J}_{\beta}(x t)+i \frac{x t^{2}}{2(\beta+1)} \mathcal{J}_{\beta+1}(x t)\right] t^{2 \beta+1}\left(1-t^{2}\right)^{\alpha-\beta-1} d t$

$=\frac{2}{B(\alpha-\beta, \beta+1)}$

$\times \int_{0}^{\frac{\pi}{2}}\left[\mathcal{J}_{\beta}(x \sin \theta)+i \frac{x(\sin \theta)^{2}}{2(\beta+1)} \mathcal{J}_{\beta+1}(x \sin \theta)\right]$

$\times(\sin \theta)^{2 \beta+1}(\cos \theta)^{2(\alpha-\beta)-1} d \theta$. 


\subsection{Concluding Remarks}

1) Let $b \in] 0, \pi[$. We have

$$
\cos b<-\frac{3 \sin (2 b)+4 b^{2} \sin b-6 b}{2\left[3 b \cos b+\sin b\left(b^{2}-3\right)\right]}<1 .
$$

a) If $|x|<x_{0}$, then

$$
\frac{1}{3}\left(\mathcal{J}_{-\frac{1}{2}}(x)+2\right)<u_{1}(b) \mathcal{J}_{-\frac{1}{2}}(x)+u_{4}(b) .
$$

b) If $x_{0}<|x| \leq \pi$, then

$$
u_{1}(b) \mathcal{J}_{-\frac{1}{2}}(x)+u_{4}(b)<\frac{1}{3}\left(\mathcal{J}_{-\frac{1}{2}}(x)+2\right) .
$$

c) If $|x|=x_{0}$, then

$$
\frac{1}{3}\left(\mathcal{J}_{-\frac{1}{2}}(x)+2\right)=u_{1}(b) \mathcal{J}_{-\frac{1}{2}}(x)+u_{4}(b),
$$

where $0<x_{0}<b$ and

$$
\cos \left(x_{0}\right):=-\frac{3 \sin (2 b)+4 b^{2} \sin b-6 b}{2\left[3 b \cos b+\sin b\left(b^{2}-3\right)\right]} .
$$

2) For all $b \in] 0, \pi[$ and $x \in[-\pi, \pi]$, we have

$u_{1}(b) \mathcal{J}_{-\frac{1}{2}}(x)+\inf \left\{1-u_{1}(b), u_{1}(b)\right\} \leq \frac{1}{2}\left(\mathcal{J}_{-\frac{1}{2}}(x)+1\right)$.

3) For all $b \in] 0, \pi[$ and $x \in \mathbb{R}$, we have

$u_{1}(b) \mathcal{J}_{-\frac{1}{2}}(x)+1-u_{1}(b) \leq \frac{1}{1-w_{1}(b)}\left(\mathcal{J}_{-\frac{1}{2}}(x)-w_{1}(b)\right)$.

4) For all $\left.b \in] 0, \frac{\pi}{2}\right]$ and $x \in \mathbb{R}$, we have

$\frac{1}{1-w_{1}(b)}\left(\mathcal{J}_{-\frac{1}{2}}(x)-w_{1}(b)\right) \leq\left(1-u_{4}(b)\right) \mathcal{J}_{-\frac{1}{2}}(x)+u_{4}(b)$.

5) For all $\left.b \in] 0, \frac{\pi}{2}\right]$ and $x \in[-\pi, \pi]$, we have

$$
\frac{1}{2}\left(\mathcal{J}_{-\frac{1}{2}}(x)+1\right) \leq\left(1-u_{4}(b)\right) \mathcal{J}_{-\frac{1}{2}}(x)+u_{4}(b) .
$$

6) For all $x \in \mathbb{R}$, we have

$$
\begin{aligned}
& \frac{1}{2}\left(\mathcal{J}_{-\frac{1}{2}}(x)+1\right) \leq \frac{1}{\pi}\left[(\pi-2) \mathcal{J}_{-\frac{1}{2}}(x)+2\right] \\
& =\left(1-u_{4}\left(\frac{\pi}{2}\right)\right) \mathcal{J}_{-\frac{1}{2}}(x)+u_{4}\left(\frac{\pi}{2}\right) .
\end{aligned}
$$

7) For all $b \in] \frac{\pi}{2}, \pi[$ and $x \in[-\pi, \pi]$, we have

$$
\frac{1}{2}\left(\mathcal{J}_{-\frac{1}{2}}(x)+1\right) \leq \frac{1}{1-w_{1}(b)}\left(\mathcal{J}_{-\frac{1}{2}}(x)-w_{1}(b)\right) .
$$

8) For all $a \in\left[\frac{\pi}{2}, \pi[\right.$ and $x \in \mathbb{R}$, we have

$$
u_{1}(a)\left(\mathcal{J}_{-\frac{1}{2}}(x)+1\right) \leq u_{4}(a)\left(\mathcal{J}_{-\frac{1}{2}}(x)+1\right) .
$$

In view of the inequalities for Bessel functions given by Á. Baricz in $[4,6]$, we can show the refinements of these inequalities as follows.

9) Let $\left.a_{1} \in\right] 0, \frac{1}{2}\left[, a_{2}:=a_{1}+1 \in\right] 1, \frac{3}{2}[$ and

$$
x \in[-\pi, \pi] \backslash\{0\} .
$$

We have

a) $0<\frac{a_{1}}{a_{2}}<\frac{1}{3}$.

b) $\frac{2}{3}<\frac{1}{a_{2}}<1$.

c) $\frac{1}{3}\left(\mathcal{J}_{-\frac{1}{2}}(x)+2\right)<\frac{a_{1}}{a_{2}} \mathcal{J}_{-\frac{1}{2}}(x)+\frac{1}{a_{2}}$.

d) $\frac{1}{3}\left(\mathcal{J}_{-\frac{1}{2}}(0)+2\right)=\frac{a_{1}}{a_{2}} \mathcal{J}_{-\frac{1}{2}}(0)+\frac{1}{a_{2}}=1$.

10) Let $\left.a_{1} \in\right] 0, \frac{1}{2}\left[, a_{3}:=\frac{\pi}{2}\right.$ and $\left.x \in\right]-\frac{\pi}{2}, \frac{\pi}{2}[$. We have

a) $0<\frac{a_{1}}{a_{3}}<\frac{1}{\pi}$.

b) $\frac{1}{a_{3}}=\frac{2}{\pi}$.

c)

$$
\begin{aligned}
& \frac{a_{1}}{a_{3}} \mathcal{J}_{-\frac{1}{2}}(x)+\frac{1}{a_{3}}<\frac{1}{\pi}\left[(\pi-2) \mathcal{J}_{-\frac{1}{2}}(x)+2\right] \\
& =\left(1-u_{4}\left(\frac{\pi}{2}\right)\right) \mathcal{J}_{-\frac{1}{2}}(x)+u_{4}\left(\frac{\pi}{2}\right) \text {. }
\end{aligned}
$$

d)

$\frac{a_{1}}{a_{3}} \mathcal{J}_{-\frac{1}{2}}\left( \pm \frac{\pi}{2}\right)+\frac{1}{a_{3}}=\frac{1}{\pi}\left[(\pi-2) \mathcal{J}_{-\frac{1}{2}}\left( \pm \frac{\pi}{2}\right)+2\right]=\frac{2}{\pi}$.

11) Let $a_{1} \in\left[\frac{1}{2}, \frac{\pi-2}{2}\left[, a_{3}:=\frac{\pi}{2}\right.\right.$ and $\left.x \in\right]-\frac{\pi}{2}, \frac{\pi}{2}[$. We have

a) $\frac{1}{\pi} \leq \frac{a_{1}}{a_{3}}<\frac{\pi-2}{\pi}$. 
b) $\frac{1}{a_{3}}=\frac{2}{\pi}$.

c) $\frac{a_{1}}{a_{3}} \mathcal{J}_{-\frac{1}{2}}(x)+\frac{1}{a_{3}}<\frac{1}{\pi}\left[(\pi-2) \mathcal{J}_{-\frac{1}{2}}(x)+2\right]$.

d)

$$
\begin{aligned}
& \frac{a_{1}}{a_{3}} \mathcal{J}_{-\frac{1}{2}}\left( \pm \frac{\pi}{2}\right)+\frac{1}{a_{3}} \\
& =\frac{1}{\pi}\left[(\pi-2) \mathcal{J}_{-\frac{1}{2}}\left( \pm \frac{\pi}{2}\right)+2\right]=\frac{2}{\pi} .
\end{aligned}
$$

12) Let $\left.a_{1} \in\right] \frac{\pi-2}{2}, \frac{2}{\pi}\left[, a_{3}:=a_{1}+1 \in\right] \frac{\pi}{2}, \frac{\pi+2}{\pi}[$ and $x \in[-\pi, \pi] \backslash\{0\}$. We have
a) $\frac{\pi-2}{\pi}<\frac{a_{1}}{a_{3}}<\frac{2}{\pi+2}$.
b) $\frac{\pi}{\pi+2}<\frac{1}{a_{3}}<\frac{2}{\pi}$.
c) ${\frac{a_{1}}{a_{3}}}_{\mathcal{J}_{-\frac{1}{2}}}(x)+\frac{1}{a_{3}}<\frac{1}{\pi}\left[(\pi-2) \mathcal{J}_{-\frac{1}{2}}(x)+2\right]$.
d) $\frac{a_{1}}{a_{3}} \mathcal{J}_{-\frac{1}{2}}(0)+\frac{1}{a_{3}}=\frac{1}{\pi}\left[(\pi-2) \mathcal{J}_{-\frac{1}{2}}(0)+2\right]=1$.

13) Let $a_{1} \geq \frac{2}{\pi}, a_{3}:=a_{1}+1 \geq \frac{\pi+2}{\pi}$ and $x \in[-\pi, \pi] \backslash\{0\}$. We have
a) $\frac{a_{1}}{a_{3}} \geq \frac{2}{\pi+2}>\frac{\pi-2}{\pi}$.
b) $\frac{1}{a_{3}} \leq \frac{\pi}{\pi+2}<\frac{2}{\pi}$.
c) $\frac{a_{1}}{a_{3}} \mathcal{J}_{-\frac{1}{2}}(x)+\frac{1}{a_{3}}<\frac{1}{\pi}\left[(\pi-2) \mathcal{J}_{-\frac{1}{2}}(x)+2\right]$.
d)

$$
\frac{a_{1}}{a_{3}} \mathcal{J}_{-\frac{1}{2}}(0)+\frac{1}{a_{3}}=\frac{1}{\pi}\left[(\pi-2) \mathcal{J}_{-\frac{1}{2}}(0)+2\right]=1 .
$$

14) Let $\alpha \geq-\frac{1}{2}$. Á. Baricz has proved in $[4,6]$ that for all $x \in\left[-\frac{\pi}{2}, \frac{\pi}{2}\right]$, we have

$$
\begin{aligned}
& \quad \frac{2(\alpha+1) a_{1} \mathcal{J}_{\alpha}(x)+1}{(2 \alpha+1) a_{1}+a_{3}} \leq \mathcal{J}_{\alpha+1}(x) \\
& \leq \frac{2(\alpha+1) a_{1} \mathcal{J}_{\alpha}(x)+1}{(2 \alpha+1) a_{1}+a_{2}},
\end{aligned}
$$

where $a_{1}, a_{2}$ and $a_{3}$ are as in Theorem 1.1 of [6]. a) If $\left.a_{1} \in\right] 0, \frac{1}{2}\left[, a_{2}:=a_{1}+1 \in\right] 1, \frac{3}{2}[$,

then $0<\frac{a_{1}}{a_{2}}<\frac{1}{3}, \frac{2}{3}<\frac{1}{a_{2}}<1$, and for all $x \in[-\pi, \pi] \backslash\{0\}$, we have

$$
\frac{(\alpha+1) \mathcal{J}_{\alpha}(x)+1}{\alpha+2}<\frac{2(\alpha+1) a_{1} \mathcal{J}_{\alpha}(x)+1}{(2 \alpha+1) a_{1}+a_{2}}
$$

and

$$
\frac{(\alpha+1) \mathcal{J}_{\alpha}(0)+1}{\alpha+2}=\frac{2(\alpha+1) a_{1} \mathcal{J}_{\alpha}(0)+1}{(2 \alpha+1) a_{1}+a_{2}}=1 .
$$

b) If $\left.a_{1} \in\right] 0,+\infty\left[\backslash\left\{\frac{\pi-2}{2}\right\}\right.$, then for all

$$
x \in[-\pi, \pi] \backslash\left\{-\frac{\pi}{2}, 0, \frac{\pi}{2}\right\},
$$

we have

$$
\frac{2(\alpha+1) a_{1} \mathcal{J}_{\alpha}(x)+1}{(2 \alpha+1) a_{1}+a_{3}}<\frac{(\alpha+1)(\pi-2) \mathcal{J}_{\alpha}(x)+1}{(\alpha+1) \pi-(2 \alpha+1)} .
$$

\subsection{Proofs}

Proof of Proposition 3.5: Let $x \in] 0, \pi[, c \in \mathbb{R}$ and

$$
u(x):=\mathcal{J}_{\frac{1}{2}}(x)-c \mathcal{J}_{-\frac{1}{2}}(x)=\frac{\sin x-c x \cos x}{x} .
$$

We have $u^{\prime}(x)=\frac{x \cos x+\left(c x^{2}-1\right) \sin x}{x^{2}}$.

$u^{\prime}(x)=0$ if and only if

$$
c=u_{1}(x):=\frac{\mathcal{J}_{\frac{3}{2}}(x)}{3 \mathcal{J}_{\frac{1}{2}}(x)}=\frac{\sin x-x \cos x}{x^{2} \sin x} .
$$

$u_{1}^{\prime}(x)=\frac{u_{2}(x)}{x^{3}(\sin x)^{2}}$, where

$$
\begin{gathered}
u_{2}(x):=x^{2}-2(\sin x)^{2}+\frac{x}{2} \sin (2 x), \\
u_{2}^{\prime}(x)=2 x+x \cos (2 x)-\frac{3}{2} \sin (2 x), \\
u_{2}^{\prime \prime}(x)=4 \sin x u_{3}(x), \\
u_{3}(x):=\frac{x^{3}}{3} j_{\frac{3}{2}}(x)=\sin x-x \cos x,
\end{gathered}
$$

$u_{3}^{\prime}(x)=x \sin x$.

$u_{2}$ is strictly increasing on $[0, \pi]$,

$$
u_{2}(0)=0<u_{2}\left(\frac{\pi}{2}\right)=\frac{\pi^{2}-8}{4}<u_{2}(\pi)=\pi^{2} .
$$


$u_{1}$ is strictly increasing on $[0, \pi]$,

$$
u_{1}(0)=\frac{1}{3}<u_{1}\left(\frac{\pi}{2}\right)=\frac{4}{\pi^{2}}, \lim _{x \rightarrow \pi^{-}} u_{1}(x)=+\infty .
$$

If $c \leq \frac{1}{3}$, then $u$ is strictly decreasing on $[0, \pi]$,

$$
u(0)=1-c \geq \frac{2}{3}>\frac{1}{3} \geq u(\pi)=c .
$$

If $c>\frac{1}{3}$, then there exists $\left.x_{1} \in\right] 0, \pi[$ such that $u_{1}\left(x_{1}\right)=c . \quad u$ is strictly increasing on $\left[0, x_{1}\right]$ and strictly decreasing on $\left[x_{1}, \pi\right]$,

$$
u(0)=1-c<u\left(x_{1}\right)=u_{4}\left(x_{1}\right), u(\pi)=c>\frac{1}{3},
$$

where

$$
u_{4}(x):=\frac{2 x-\sin (2 x)}{2 x^{2} \sin x}, u_{4}^{\prime}(x)=-\frac{\cos x u_{2}(x)}{x^{3}(\sin x)^{2}} .
$$

$u_{4}$ is strictly decreasing on $\left[0, \frac{\pi}{2}\right]$ and strictly increasing on $\left[\frac{\pi}{2}, \pi[\right.$,

$$
u_{4}(0)=\frac{2}{3}, u_{4}\left(\frac{\pi}{2}\right)=\frac{2}{\pi}, \lim _{x \rightarrow \pi^{-}} u_{4}(x)=+\infty .
$$

Proof of Proposition 3.6: Let $x \in] 0, \pi\left[\backslash\left\{\frac{\pi}{2}\right\}, c \in \mathbb{R}\right.$ and

$$
v(x):=\frac{\mathcal{J}_{\frac{1}{2}}(x)-c}{\mathcal{J}_{-\frac{1}{2}}(x)}=\frac{\sin x-c x}{x \cos x} .
$$

We have $v^{\prime}(x)=\frac{2 x-\sin (2 x)-2 c x^{2} \sin x}{2 x^{2}(\cos x)^{2}}$.

$v^{\prime}(x)=0$ if and only if $c=u_{4}(x)$, where $u_{4}$ is given by (5).

If $c<\frac{2}{\pi}$, then $v$ is strictly increasing on $\left[0, \frac{\pi}{2}[[0, \pi]\right.$ and $\left.] \frac{\pi}{2}, \pi\right], \frac{\pi-2}{\pi}<v(0)=1-c, \quad v(\pi)=c<\frac{2}{\pi}$,

$$
\lim _{x \rightarrow\left(\frac{\pi}{2}\right)^{-}} v(x)=+\infty, \quad \lim _{x \rightarrow\left(\frac{\pi}{2}\right)^{+}} v(x)=-\infty
$$

If $c=\frac{2}{\pi}$, then $v$ is strictly increasing on $[0, \pi]$,

$$
v(0)=\frac{\pi-2}{\pi}<v\left(\frac{\pi}{2}\right)=\frac{4}{\pi^{2}}<v(\pi)=\frac{2}{\pi} .
$$

If $c \geq \frac{2}{3}$, then there exist $a_{0}, x_{1} \in \mathbb{R}$ such that

$$
\frac{\pi}{2}<a_{0} \leq x_{1}<\pi, u_{4}\left(a_{0}\right):=\frac{2}{3}, u_{4}\left(x_{1}\right)=c .
$$

$v$ is strictly decreasing on $\left[0, \frac{\pi}{2}[\right.$ and $\left.] \frac{\pi}{2}, x_{1}\right]$, and strictly increasing on $\left[x_{1}, \pi\right]$,

$$
\begin{gathered}
v(0)=1-c, \\
\frac{4}{\pi^{2}}<u_{1}\left(a_{0}\right) \leq v\left(x_{1}\right)=u_{1}\left(x_{1}\right)<v(\pi)=c, \\
\lim _{x \rightarrow\left(\frac{\pi}{2}\right)^{-}} v(x)=-\infty, \quad \lim _{x \rightarrow\left(\frac{\pi}{2}\right)^{+}} v(x)=+\infty,
\end{gathered}
$$

where $u_{1}$ is given by (4).

If $c \in] \frac{2}{\pi}, \frac{2}{3}\left[\right.$, then there exist $x_{2}, x_{3} \in \mathbb{R}$ such that $0<x_{2}<\frac{\pi}{2}<x_{3}<a_{0}, u_{4}\left(x_{2}\right)=u_{4}\left(x_{3}\right)=c . \quad v$ is strictly increasing on $\left[0, x_{2}\right]$ and $\left[x_{3}, \pi\right]$, and strictly decreasing on $\left[x_{2}, \frac{\pi}{2}[\right.$ and $\left.] \frac{\pi}{2}, x_{3}\right]$,

$$
\begin{gathered}
\frac{1}{3}<v(0)=1-c<v\left(x_{2}\right)=u_{1}\left(x_{2}\right), \\
\frac{4}{\pi^{2}}<v\left(x_{3}\right)=u_{1}\left(x_{3}\right)<v(\pi)=c, \\
\lim _{x \rightarrow\left(\frac{\pi}{2}\right)^{-}} v(x)=-\infty, \quad \lim _{x \rightarrow\left(\frac{\pi}{2}\right)^{+}} v(x)=+\infty .
\end{gathered}
$$

Proof of Proposition 3.7: Let $x \in] 0, \pi[, c \in \mathbb{R}$ and

$$
w(x):=\frac{\mathcal{J}_{-\frac{1}{2}}(x)-c}{\mathcal{J}_{\frac{1}{2}}(x)}=\frac{x(\cos x-c)}{\sin x} .
$$

We have

$$
w^{\prime}(x)=-\frac{2 x-\sin (2 x)+2 c(\sin x-x \cos x)}{2(\sin x)^{2}} .
$$

$w^{\prime}(x)=0$ if and only if $c=w_{1}(x):=-\frac{2 x-\sin (2 x)}{2(\sin x-x \cos x)}$. $w_{1}^{\prime}(x)=\frac{\sin x u_{2}(x)}{(\sin x-x \cos x)^{2}}$, where $u_{2}$ is given by (7). $w_{1}$ is strictly increasing on $[0, \pi]$,

$$
w_{1}(0)=-2<w_{1}\left(\frac{\pi}{2}\right)=-\frac{\pi}{2}<w_{1}(\pi)=-1 .
$$

If $c \leq-2$, then $w$ is strictly increasing on $[0, \pi[$,

$$
3 \leq w(0)=1-c<w\left(\frac{\pi}{2}\right)=-\frac{c \pi}{2},
$$

$$
\lim _{x \rightarrow \pi^{-}} w(x)=+\infty \text {. }
$$


If $c>-1$, then $w$ is strictly increasing on $[0, \pi[$,

$$
\begin{gathered}
2>w(0)=1-c>w\left(\frac{\pi}{2}\right)=-\frac{c \pi}{2}, \\
\lim _{x \rightarrow \pi^{-}} w(x)=-\infty .
\end{gathered}
$$

If $c=-1$, then $w$ is strictly increasing on $[0, \pi]$,

$$
w(0)=2>w\left(\frac{\pi}{2}\right)=\frac{\pi}{2}>w(\pi)=0 .
$$

If $c \in]-2,-1\left[\right.$, then there exists $\left.x_{1} \in\right] 0, \pi[$ such that $w_{1}\left(x_{1}\right)=c$.

$w$ is strictly decreasing on $\left[0, x_{1}\right]$ and strictly increasing on $\left[x_{1}, \pi[\right.$,

$$
\begin{gathered}
3>w(0)=1-c>w\left(x_{1}\right)=\frac{1}{u_{1}\left(x_{1}\right)}>0, \\
\lim _{x \rightarrow \pi^{-}} w(x)=+\infty,
\end{gathered}
$$

where $u_{1}$ is given by (4).

Proof of Proposition 3.8: Let $x \in \mathbb{R} \backslash\{0\}$ and

$$
d(x):=\mathcal{J}_{\frac{3}{2}}(x)=3 \frac{\sin x-x \cos x}{x^{3}} .
$$

We have $d^{\prime}(x)=\frac{3 d_{1}(x)}{x^{4}}$,

$$
\begin{gathered}
d_{1}(x):=3 x \cos x+\left(x^{2}-3\right) \sin x, \\
d_{1}^{\prime}(x)=-x d_{2}(x), d_{2}(x):=\sin x-x \cos x, \\
d_{2}^{\prime}(x)=x \sin x .
\end{gathered}
$$

$d_{1}$ is strictly decreasing on $[-\pi, \pi]$,

$$
d_{1}(-\pi)=3 \pi>d_{1}(0)=0>d_{1}(\pi)=-3 \pi .
$$

$d$ is even on $\mathbb{R}$ and strictly increasing on $[-\pi, 0]$ and strictly decreasing on $[0, \pi]$,

$$
d(0)=1>d\left( \pm \frac{\pi}{2}\right)=\frac{24}{\pi^{3}}>d( \pm \pi)=\frac{3}{\pi^{2}} .
$$

Proof of Theorem 3.1: We get the result from Propositions 3.5, 3.6, 3.7 and Remarks $1, \ldots, 8$.

Proof of Theorem 3.2: Let $\alpha>-\frac{1}{2}$,

$$
\left.l_{1}, r_{1} \in\right]-\frac{1}{2 \alpha+1},+\infty\left[, \quad l_{2}, r_{2} \in \mathbb{R}\right.
$$

and $x \in I=[-b, b], 0<b \leq \pi$.

$$
\text { If } l_{1} \mathcal{J}_{-\frac{1}{2}}(x)+l_{2} \leq \mathcal{J}_{\frac{1}{2}}(x) \leq r_{1} \mathcal{J}_{-\frac{1}{2}}(x)+r_{2} \text {, then by using }
$$

Proposition 3.9 and

$$
B\left(\alpha+\frac{1}{2}, \frac{3}{2}\right)=\frac{B\left(\alpha+\frac{1}{2}, \frac{1}{2}\right)}{2(\alpha+1)}=\frac{B\left(\alpha+\frac{3}{2}, \frac{1}{2}\right)}{2 \alpha+1},
$$

we deduce

$$
\begin{aligned}
& \frac{2(\alpha+1) l_{1} \mathcal{J}_{\alpha}(x)+l_{2}}{(2 \alpha+1) l_{1}+1} \leq \mathcal{J}_{\alpha+1}(x) \\
& \leq \frac{2(\alpha+1) r_{1} \mathcal{J}_{\alpha}(x)+r_{2}}{(2 \alpha+1) r_{1}+1} .
\end{aligned}
$$

Theorem 3.1 finishes the proof.

Proof of Theorem 3.4: Let $\alpha>-\frac{1}{2}$,

$$
\left.l_{1}, r_{1} \in\right]-\frac{3}{2 \alpha+1},+\infty\left[, \quad l_{2}, r_{2} \in \mathbb{R}\right.
$$

and $x \in I=[0, b], 0<b \leq \pi$.

If $l_{1} \mathcal{J}_{\frac{1}{2}}(x)+l_{2} \leq \mathcal{J}_{\frac{3}{2}}(x) \leq r_{1} \mathcal{J}_{\frac{1}{2}}(x)+r_{2}$, then by using

Proposition 3.9 and

$$
B\left(\alpha+\frac{1}{2}, \frac{3}{2}\right)=\frac{B\left(\alpha+\frac{1}{2}, \frac{1}{2}\right)}{2(\alpha+1)}=\frac{B\left(\alpha+\frac{3}{2}, \frac{1}{2}\right)}{2 \alpha+1},
$$

we deduce

$$
\begin{aligned}
& \frac{2(\alpha+1) l_{1} \mathfrak{J} \psi_{1}^{\alpha}(x)+l_{2} x}{(2 \alpha+1) l_{1}+3} \leq \mathfrak{I} \psi_{1}^{\alpha+1}(x) \\
& \leq \frac{2(\alpha+1) r_{1} \mathfrak{J} \psi_{1}^{\alpha}(x)+r_{2} x}{(2 \alpha+1) r_{1}+3} .
\end{aligned}
$$

Theorem 3.2 with $\alpha=\frac{1}{2}$ finishes the proof.

\section{Acknowledgements}

Thanks to the referee for careful reading and helpful comments.

\section{References}

[1] Carver, W.B. and Oppenheim, A., Elementary problems and solutions: solutions: E 1277, Amer. Math. Monthly, 65(3). 206209. 1958.

[2] Mitrinović, D.S., Analytic inequalities, Springer-Verlag, Berlin, 1970.

[3] Zhu, L., A solution of a problem of Oppenheim. Math. Inequal. Appl., 10(1). 57-61. 2007.

[4] Baricz, Á., Functional inequalities involving Bessel and modified Bessel functions of the first kind, Expo. Math., 26(3). 279-293. 2008.

[5] Chouchene, F., Oppenheim's problem and related inequalities for Dunkl kernels, Math. Inequal. Appl., 17(1). 1-40. 2014.

[6] Baricz, Á. and Zhu, L., Extension of Oppenheim's problem to Bessel functions. J. Inequal. Appl., Art. ID 82038.7 pp. 2007.

[7] Baricz, Á., Generalized Bessel functions of the first kind, Lecture Notes in Mathematics, 1994. Springer-Verlag, Berlin, 2010. xiv+206 pp.

[8] Chettaoui, C. and Trimèche, K., New type Paley-Wiener theorems for the Dunkl transform on $\mathbb{R}$. Integral Transforms Spec. Funct., 14(2). 97-115. 2003.

[9] Dunkl, C.F., Differential-difference operators associated to reflection groups. Trans. Amer. Math. Soc., 311(1). 167-183. 1989

[10] Dunkl, C.F., Integral kernels with reflection group invariance, Canad. J. Math., 43. 1213-1227. 1991. 
[11] Dunkl, C.F., Hankel transforms associated to finite reflection groups, Contemp. Math., 138. 123-138. 1992.

[12] Dunkl, C.F., Intertwining operators and polynomials associated with the symmetric group, Monatsh. Math., 126. 181-209. 1998.

[13] Dunkl, C.F., Orthogonal polynomials of types A and B and related Calegero models, Commun. Math. Phys., 197. 451-487. 1998.

[14] Mourou, M.A., Transmutation operators associated with a Dunkl type differential-difference operator on the real line and certain of their applications, Integral Transforms Spec. Funct., 12(1). 77-88. 2001.

[15] Mourou, M.A. and Trimèche, K., Opérateurs de transmutation et théorème de Paley-Wiener associés à un opérateur aux dérivées et différences sur $\mathbb{R}$, C. R. Acad. Sci. Paris, Série I Math., 332. $397-$ 400. 2001.

[16] Mourou, M.A. and Trimèche, K., Transmutation operators and Paley-Wiener theorem associated with a differential-difference operator on the real line, Anal. Appl., 1. 43-70. 2003.

[17] Rösler, M., Bessel-type signed hypergroups on $\mathbb{R}$. In: H. Heyer, A. Mukherjea (eds.). Probability measures on groups and related structures XI. Proceedings. Oberwolfach 1994, Singapore: World Scientific, 292-304. 1995.

[18] Watson, G.N., A treatise on the theory of Bessel functions, Cambridge University Press, Cambridge, UK, 1962. 